\section{A Meta-analysis of Strawberry Yield Response to Preplant Soil Fumigation with Combinations of Methyl Bromide- chloropicrin and Four Alternative Systems}

\author{
Douglas V. Shaw and Kirk D. Larson \\ Pomology Department, University of California, Davis, CA 95616
}

Additional index words. chloropicrin, dichloropropene, Fragaria $\times$ ananassa, metam sodium, methyl bromide

\begin{abstract}
Yield for annual California strawberry (Fragaria $\times$ ananassa Duch.) production systems in soils treated with combinations of methyl bromide-chloropicrin (MB:CP) were compared with four alternative soil treatment systems using meta-analysis. Studies represent 11 production seasons, and were conducted at three distinct locations in California. Fumigation with mixtures of methyl bromide (MB) and chloropicrin (CP) increased yield significantly compared with any and all alternatives lacking MB. In a combined analysis of 45 studies, fumigation with MB:CP compounds increased yield an average of $94.4 \%\left(d_{+}=2.874 \pm 0.098\right)$ compared with yields for plants in nonfumigated $(\mathrm{NF})$ soils. Further, the effect of MB:CP fumigation increased over the first three strawberry cultivation cycles: MB:CP-fumigated soils provided a 59.2\% $\left(d_{+}=2.166 \pm 0.146\right)$ yield advantage when one cycle of fumigation was omitted, a $100.2 \%\left(d_{+}=3.000 \pm 0.143\right)$ advantage when two cycles were omitted, and a $148.4 \%\left(d_{+}=6.201 \pm 0.348\right)$ yield advantage when three or more cycles of MB:CP were omitted. In a combined analysis that included 34 studies, soil fumigation with MB:CP conferred a 9.6\% $\left(d_{+}=0.751 \pm 0.087\right)$ yield advantage over fumigation with $\mathrm{CP}$ alone. Soils treated with MB:CP yielded $6.8 \%\left(d_{+}=\right.$ $0.437 \pm 0.114)$ more fruit than those treated with very high rates of $C P\left(336-396 \mathrm{~kg}^{\circ} \cdot \mathrm{ha}^{-1}\right)$, and $15.4 \%\left(d_{+}=1.190 \pm 0.134\right)$ more than soils treated with commercially realistic rates $\left(168-224 \mathrm{~kg} \cdot \mathrm{ha}^{-1}\right)$. Similar to the comparison using NF soils, the efficacy of very high rates of CP appeared to diminish over cycles of strawberry cultivation; $\mathrm{MB}$ : $\mathrm{CP}$ increased yield $2.2 \%\left(d_{+}=0.043 \pm 0.162\right)$ in the first $C P$ production cycle, $10.6 \%\left(d_{+}=0.588 \pm 0.174\right)$ and $13.7 \%\left(d_{+}=2.054 \pm 0.401\right)$ in the following two cycles. Combinations of dichloropropene (DP) and CP were no more effective than were lower rates of $\mathrm{CP}$ alone, and MB:CP conferred a $14.4 \%\left(d_{+}=0.962 \pm 0.162\right)$ yield advantage over mixtures of DP:CP. Mixtures of MB:CP increased yield $29.8 \%\left(d_{+}=3.199 \pm 0.287\right)$ compared with metam sodium (MS). The standardized effect was similar when comparing MB:CP combinations with either MS or NF soils, suggesting little effect of MS on the yield response. Chemical names used: trichloronitromethane (chloropicrin); 1,3-dichloropropene (dichloropropene); sodium $N$-methyldithiocarbamate (metam sodium).
\end{abstract}

Preplant soil fumigation was originally investigated as a means for controlling Verticillium dahliae Koch (Wilhelm and Koch, 1956) in strawberry. Subsequent studies demonstrated advantages of preplant soil fumigation for at least three horticultural issues: weed control, control of lethal pathogens and nematodes, and a general growth and yield response that appears independent of specific hostpathogen interactions (Wilhelm et al., 1961, 1974). Studies of a range of other fumigation alternatives generally concluded that a synergistic effect of compounds containing methyl

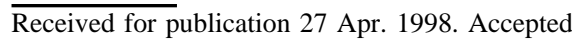
for publication 5 Feb. 1999. We thank A. Paulus, D. Walsh, N. Welch, and F. Zalom for contributing unpublished results to this research synthesis. The cost of publishing this paper was defrayed in part by the payment of page charges. Under postal regulations, this paper therefore must be hereby marked advertisement solely to indicate this fact.

HortScience, Vol. 34(5), August 1999 tially from minimal treatment such as repeated use of nonfumigated (NF) soils (with or without crop rotation) to application of alternative chemicals at high rates, in an effort to quantify the consequences of a ban on MB. This synthesis is limited in scope in that it does not consider the consequences of lethal pathogens that are likely to be a greater problem with some of the available alternatives. Further, this analysis does not quantify reductions in fruit quality, economic losses due to the cost of alternative chemicals or fruit quality reduction, or capital costs and/or yield reductions that might accrue as a result of any changes in cultural practice changes required by alternatives to MB:CP. This synthesis also considers only yield effects for soil treatments applied in the fruiting field. Strawberry production systems depend on preplant soil fumigation in a number of nursery propagation steps, and preliminary indications suggest that problems may arise for both plant productivity and quality using available alternatives (Larson and Shaw, 1995b). The effects of eliminating MB on nursery production, plant quality, and eventual yield reduction due to reduced nursery plant quality will be considered separately. This synthesis begins by defining strawberry performance in the NF control. Thereafter, the choice of comparison treatments was limited to those that had demonstrated some promise in prior experiments, and to chemical alternatives currently registered and thus available for use in California. As a consequence, several chemical and nonchemical alternatives that had either shown little effect in prior trials or are not currently registered in California were not included. Lastly, several of the chemical alternatives were tested at rates too high to be legally, economically, or socially feasible in operational production systems; these trials were included to provide boundaries for efficacy estimates.

\section{Materials and Methods}

The individual studies used for this research synthesis were conducted at three macro-locations within California: Irvine, at the south edge of the Los Angeles basin $\left(33^{\circ} 40^{\prime}\right.$, $117^{\circ} 43^{\prime}$ ); Oxnard, $\approx 97 \mathrm{~km}$ north of Los Angeles $\left(34^{\circ} 13^{\prime}, 119^{\circ} 11^{\prime}\right)$; and Watsonville, $\approx 130$ $\mathrm{km}$ south of San Francisco (36 $56^{\prime}, 121^{\circ} 46^{\prime}$ ). These locations are representative of the environments that produce $>97 \%$ of the strawberries grown in California, or $\approx 80 \%$ of those produced in North America (Faxon, 1997). Results were obtained from trials conducted on three subsites in the Irvine region, two in the Oxnard region, and three in the Watsonville region.

The trials included in this synthesis extend from 1987 to 1997 ; most were conducted after 1992, as a consequence of increasing concerns about future availability of MB. Fumigation trials prior to 1987 frequently used germplasm no longer considered commercially viable, a potentially confounding factor eliminated in this synthesis.

Most of the trials were conducted using commercial cultivars adapted to the specific 
test region chosen, although a few comparisons included advanced selection materials from the Univ. of California (UC) strawberry improvement program, and two of the NF trials used cultivars of other North American origin. Likewise, several of the studies included runner plants from nurseries treated with different fumigants. Where multiple cultivars were used or where several nursery fumigation treatments were applied, the standard deviations used for weighting in combined analysis were calculated using pooled within-genotype or within-nursery-treatment sums of squares.

The results presented in this synthesis represent a reasonably complete array of those studies conducted to test fumigation alternatives by public sector researchers in California between 1987 and 1997. Original results requested from two authors for four studies were no longer available, and because the published results lacked the necessary parameters (means, SDs, sample numbers) for inclusion in a metaanalysis, these were omitted. Also, results for six studies were judged proprietary by their originators and not made available for this synthesis. The vast majority of research results synthesized here were reported in symposia, industry bulletins, or are unpublished at present because of recent completion; these publications rarely included the necessary parameters for inclusion in a meta-analysis. In these cases, parameters were obtained from raw data provided by the original authors and reanalyzed for this synthesis. Typically, individual studies contained an industry standard control (fumigated with $\mathrm{MB}: \mathrm{CP}$ ), a NF control, and one or more chemical alternatives. Individual comparisons were made between the industry standard treatment $(\mathrm{MB}: \mathrm{CP})$ and each of several different alternatives within a single trial. Thus, results from the MB:CP treatment were used in multiple comparisons. As a consequence, comparisons of the different alternatives are not independent, but their covariance is expected to be large only when very small numbers of studies are evaluated, and thus will be of little importance here.

Study results were combined following the meta-analysis procedures of Hedges and Olkin (1985), as summarized in Olkin and Shaw (1995). Meta-analysis refers to a set of statistical techniques developed to provide a quantitative comparison of research results obtained from many independent studies. These techniques adjust for differences in error rate among studies and weight individual results based on both intrinsic error and sample size. In our synthesis, yield differences for treated and control groups in individual studies were converted to standardized effects, $d$, as:

$$
\mathrm{d}=\frac{\overline{\mathrm{X}}_{\mathrm{T}}-\overline{\mathrm{X}}_{\mathrm{C}}}{\mathrm{s}}
$$

$\overline{\mathrm{X}}_{\mathrm{T}}$ and $\overline{\mathrm{X}}_{\mathrm{C}}$ are means for the treated and control groups, respectively, and sis the pooled standard deviation for both groups. To obtain a composite estimate for the magnitude of a given treatment effect, the $\mathrm{d}_{\mathrm{i}}$ 's from individual studies were weighted according to individual study error rates, $\hat{\boldsymbol{O}}^{2}\left(\mathrm{~d}_{\mathrm{i}}\right)$, where:

$$
\sigma^{2}\left(d_{i}\right)=\frac{n_{i}^{T}+n_{i}^{C}}{n_{i}^{T} n_{i}^{C}}+\frac{d_{i}^{2}}{2\left(n_{i}^{T}+n_{i}^{C}\right)}
$$

In Eq. $2, \mathrm{n}_{\mathrm{i}}^{\mathrm{T}}$ and $\mathrm{n}_{\mathrm{i}}^{\mathrm{C}}$ are the numbers of replications for treated and control groups, respectively, and $d_{i}$ is the estimated treatment effect for the $i^{\text {th }}$ study, $i=1, \ldots, k$. The weight, $\mathrm{w}_{\mathrm{i}}$, assigned to each effect size estimate is inversely proportional to its variance and was computed as:

$$
\mathrm{w}_{\mathrm{i}}=\frac{1 / \hat{\sigma}^{2}\left(\mathrm{~d}_{\mathrm{i}}\right)}{\sum\left[1 / \hat{\sigma}^{2}\left(\mathrm{~d}_{\mathrm{j}}\right)\right]}
$$

and the combined estimate, $d_{+}$, of effect size was calculated as:

$$
\mathrm{d}_{+}=\mathrm{w}_{1} \mathrm{~d}_{1}+\mathrm{w}_{2} \mathrm{~d}_{2}+\ldots+\mathrm{w}_{\mathrm{k}} \mathrm{d}_{\mathrm{k}}=\sum \mathrm{w}_{\mathrm{i}} \mathrm{d}_{\mathrm{i}}
$$

Statistical comparisons were made using 95\% confidence intervals constructed from the estimated variance of the combined effect size, $\hat{\boldsymbol{\sigma}}\left(\mathrm{d}_{+}\right)$, calculated as:

$$
\hat{\sigma}^{2}\left(d_{+}\right)=\frac{1}{\Sigma\left[1 / \hat{\sigma}^{2}\left(d_{j}\right)\right]}
$$

A confidence interval for the combined effect size was determined as $d_{+} \pm c_{\alpha / 2} \hat{\sigma}\left(d_{+}\right)$, with $\mathrm{c}_{\alpha / 2}$ being the appropriate coefficient obtained from the normal distribution for the chosen confidence interval; for example, 1.96 for a 95\% confidence interval.

Sufficient numbers of studies were obtained to compare four alternatives with the $\mathrm{MB}: \mathrm{CP}$ industry standard: a NF control, CP alone at high and moderate rates, mixtures of dichloropropene (DP) and CP, and metam sodium (MS). In all cases, the critical comparison was between the current industry standard and an alternative, with $\mathrm{MB}: \mathrm{CP}$ designated as the treated group and one of the alternatives listed above as the control. Thus, the standardized effect sizes provided by metaanalysis, statistical comparisons based on their confidence limits, and the unweighted percentage increase in yield provide a direct comparison between efficacy of fumigation using $\mathrm{MB}: \mathrm{CP}$ and that of each of the designated alternatives. Figures are expressed in standardized effect size or percentage advantage for the current fumigation system, but could be expressed as relative losses for the alternatives by simple transformation.

Because most trials included both an industry standard and a NF control, comparison between fumigation with $\mathrm{MB}: \mathrm{CP}$ mixtures and the absence of fumigation is the best tested of the treatment effects evaluated here; 45 studies were available for this comparison (Table 1, study set "N" in the far right column). Trials with NF included both those that followed strawberry cultivation directly with another strawberry crop and those that include a crop rotation other than strawberry. Prior work showed little difference in fumigation response with either 1 or 20 years rotation out of strawberry (Larson and Shaw, 1995a). Similarly, no mean difference was apparent among sets of studies established with and without crop rotations consisting of alternate years of strawberry and a barley (Hordeum vulgare L.)/fava bean (Vicia faba L.) cover crop that was incorporated into the soil every other spring, thus any rotation effect was ignored. Trials differed in the number of strawberry production cycles since the last fumigation cycle, and we subdivided our NF studies into sets with one, two, or more than two strawberry cycles to evaluate the changes in relative productivity over time.

Direct comparison of yield for plants in soils fumigated with $\mathrm{MB}: \mathrm{CP}$ mixtures and those grown in soils fumigated with $\mathrm{CP}$ alone were possible using 34 studies (Table 1, study set " $C$ " in the far right column). Twenty-three of these studies were conducted using $\mathrm{CP}$ at rates of $336 \mathrm{~kg} \cdot \mathrm{ha}^{-1}$ or greater; due to the cost of materials and the social consequences of emissions of this compound, such rates are not likely to be commercially realistic. Eleven studies were conducted using moderate, and more realistic, rates $\left(121-224 \mathrm{~kg} \cdot \mathrm{ha}^{-1}\right)$. Rate differences were compared in a subdivided meta-analysis. The effect of high rates of $\mathrm{CP}$ on yield was also evaluated by subdividing the 23 high-rate studies over the number of cycles since the last fumigation with $\mathrm{MB}: \mathrm{CP}$ combinations; a similar subdivision was not possible for $\mathrm{CP}$ at moderate rates due to an insufficient number of studies.

Ten studies were available for comparing yields of plants grown in soils treated with MB:CP vs. DP:CP mixtures (Table 1, study set " $D$ " in the far right column). These 10 studies are a heterogeneous mix with substantial differences in the total quantity of product applied (336-666 kg.ha ${ }^{-1}$ ) and, importantly, from $94-235 \mathrm{~kg} \cdot \mathrm{ha}^{-1}$ of $\mathrm{CP}$ as part of the mixture. Several of the studies used DP at rates that exceed the maximum currently allowed by regulatory agencies.

Eight studies were included in yield comparisons for MB:CP vs. MS (Table 1, study set " $M$ " in the far right column). Seven of the eight studies were conducted using the recommended rates of $275-305 \mathrm{~L} \cdot \mathrm{ha}^{-1}$ a.i of MS, the other used $153 \mathrm{~L} \cdot \mathrm{ha}^{-1}$ a.i. The treatment differences were somewhat larger for this comparison, and comparisons were rather precise despite inclusion of relatively few studies. Fewer studies were available for evaluating the effect of MS on strawberry yield during the past 11 seasons, probably because a large number of prior studies had routinely demonstrated this to be a less effective soil treatment (Himelrick and Dozier, 1991). Three studies not included because they lacked appropriate statistical information will be discussed to support these comparisons.

\section{Results}

Yields were larger following fumigation with $\mathrm{MB}: \mathrm{CP}$ than for $\mathrm{NF}$ treatments in all studies, and these differences were statistically significant or highly significant in 41 of the 45 individual studies evaluated (Table 2). Over all studies the weighted standardized effect was $d_{+}=2.874 \pm 0.098(95 \%$ confidence: $2.672,3.056$ ), indicating a significant 


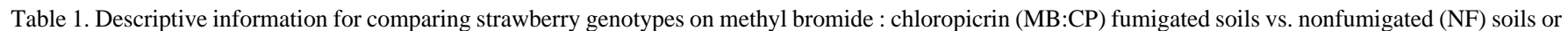
on soils treated with chloropicrin (CP), dichloropropene: chloropicrin (DP:CP), or metam sodium.

\begin{tabular}{|c|c|c|c|c|c|c|c|c|}
\hline $\begin{array}{l}\text { Study } \\
\text { no. }\end{array}$ & $\begin{array}{l}\text { Test } \\
\text { year }\end{array}$ & Location & $\begin{array}{l}\text { Cultivars/ } \\
\text { selections }\end{array}$ & Crop & Treatment $^{2}$ & Rate/ha & Reference & $\begin{array}{c}\text { Study } \\
\text { set }^{y} \\
\end{array}$ \\
\hline 1 & 1987 & Watsonville & Chandler & First NF & $\mathrm{NF}$ & --- & Welch, unpublished & $\mathrm{N}$ \\
\hline 2 & 1988 & Watsonville & Chandler & $\begin{array}{l}\text { First DP:CPx } \\
\text { and NF }\end{array}$ & $\begin{array}{l}\text { NF } \\
83 \mathrm{DP}: 17 \mathrm{CP}\end{array}$ & $666 \mathrm{~kg}$ & Welch, unpublished & N,D \\
\hline 3 & 1989 & Watsonville & Chandler & Second NF & $\mathrm{NF}$ & --- & Welch et al., 1990 & $\mathrm{~N}$ \\
\hline 4 & 1989 & Watsonville & Chandler & Second NF & $\mathrm{NF}$ & --- & Welch et al., 1990 & $\mathrm{~N}$ \\
\hline 5 & 1990 & Watsonville & Chandler & $\begin{array}{l}\text { Third DP:CP } \\
\text { and NF }\end{array}$ & $\begin{array}{l}\text { NF } \\
83 \mathrm{DP}: 17 \mathrm{CP}\end{array}$ & $666 \mathrm{~kg}$ & Welch et al., 1991 & $\mathrm{~N}, \mathrm{D}$ \\
\hline 6 & 1990 & Watsonville & Chandler & Third NF & $\mathrm{NF}$ & --- & Welch et al., 1991 & $\mathrm{~N}$ \\
\hline 7 & 1991 & Watsonville & Selva & $\begin{array}{l}\text { First DP:CPx } \\
\text { and NF }\end{array}$ & $\begin{array}{l}\text { NF } \\
83 \mathrm{DP}: 17 \mathrm{CP}\end{array}$ & $554 \mathrm{~kg}^{2}$ & Welch, unpublished & $\mathrm{N}, \mathrm{D}$ \\
\hline 8 & 1992 & Watsonville & Selva & First $\mathrm{MS}^{\mathrm{x}}$ & MS & $842 \mathrm{~L}$ & Welch, unpublished & M \\
\hline 9 & 1993 & Watsonville & $\begin{array}{l}12 \text { UC } \\
\text { cultivars/ } \\
\text { selections }\end{array}$ & First NF & $\mathrm{NF}$ & --- & Larson and Shaw, 1994 & \\
\hline 10 & 1993 & Irvine & $\begin{array}{l}12 \text { UC } \\
\text { cultivars/ } \\
\text { selections }\end{array}$ & First NF & NF & --- & Larson and Shaw, 1994 & $\mathrm{~N}$ \\
\hline 11 & 1993 & Irvine & Chandler & $\begin{array}{l}\text { First } \mathrm{CP} \text { and NF } \\
\text { crop, 20-yr } \\
\text { rotation }\end{array}$ & $\begin{array}{l}\mathrm{NF} \\
\mathrm{CP}\end{array}$ & $112 \mathrm{~kg}$ & $\begin{array}{l}\text { Larson and Sterrett, } \\
1994\end{array}$ & $\mathrm{~N}, \mathrm{C}$ \\
\hline 12 & 1993 & Irvine & $\begin{array}{l}\text { Oso } \\
\text { Grande }\end{array}$ & $\begin{array}{l}\text { First } \mathrm{CP} \text { and NF, } \\
20-\mathrm{yr} \text { rotation }\end{array}$ & $\begin{array}{l}\mathrm{NF} \\
\mathrm{CP}\end{array}$ & $112 \mathrm{~kg}$ & $\begin{array}{l}\text { Larson and Sterrett, } \\
1994\end{array}$ & $\mathrm{~N}, \mathrm{C}$ \\
\hline 13 & 1993 & Watsonville & $\begin{array}{l}\text { four UC } \\
\text { cultivars }\end{array}$ & Second NF & NF & --- & Walsh et al., 1996 & $\mathrm{~N}$ \\
\hline 14 & 1994 & Irvine & Camarosa & Second NF & NF & --- & Larson, unpublished & $\mathrm{N}$ \\
\hline 15 & 1994 & Irvine & $\begin{array}{l}\text { six UC } \\
\text { cultivars }\end{array}$ & $\begin{array}{l}\text { Second CP and NF } \\
\mathrm{CP}\end{array}$ & $\begin{array}{l}\mathrm{NF} \\
336 \mathrm{~kg}\end{array}$ & --- & Larson, unpublished & $\mathrm{N}, \mathrm{C}$ \\
\hline 16 & 1994 & Irvine & Chandler & $\begin{array}{l}\text { Second } \mathrm{CP} \text { and } \mathrm{NF} \\
\mathrm{CP}\end{array}$ & $\begin{array}{l}\mathrm{NF} \\
336 \mathrm{~kg}\end{array}$ & --- & Larson et al., 1996 & $\mathrm{~N}, \mathrm{C}$ \\
\hline 17 & 1994 & Watsonville & Selva & $\begin{array}{l}\text { First } \mathrm{CP} \text { and NF } \\
\mathrm{CP}\end{array}$ & $\begin{array}{l}\mathrm{NF} \\
336 \mathrm{~kg}\end{array}$ & --- & Larson, unpublished & $\mathrm{N}, \mathrm{C}$ \\
\hline 18 & 1994 & Watsonville & Selva & $\begin{array}{l}\text { First } \mathrm{CP} \text { and NF } \\
\mathrm{CP}\end{array}$ & $\begin{array}{l}\mathrm{NF} \\
336 \mathrm{~kg}\end{array}$ & --- & Larson, unpublished & $\mathrm{N}, \mathrm{C}$ \\
\hline 19 & 1994 & Watsonville & Selva & $\begin{array}{l}\text { First } \mathrm{CP} \text { and NF } \\
\mathrm{CP}\end{array}$ & $\begin{array}{l}\mathrm{NF} \\
336 \mathrm{~kg}\end{array}$ & --- & Larson, unpublished & $\mathrm{N}, \mathrm{C}$ \\
\hline 20 & 1994 & Watsonville & Selva & $\begin{array}{l}\text { First } \mathrm{CP} \text { and } \mathrm{NF} \\
\mathrm{CP}\end{array}$ & $\begin{array}{l}\text { NF } \\
336 \mathrm{~kg}\end{array}$ & --- & Larson, unpublished & $\mathrm{N}, \mathrm{C}$ \\
\hline 21 & 1994 & Oxnard & Chandler & $\begin{array}{l}\text { First DP:CPx }, \mathrm{CP}^{\mathrm{x}} \text {, } \\
\mathrm{MS}^{\mathrm{x}} \text { and NF}\end{array}$ & $\begin{array}{l}\mathrm{NF} \\
\mathrm{CP} \\
7 \mathrm{DP}: 3 \mathrm{CP}\end{array}$ & $\begin{array}{l}--- \\
396 \mathrm{~kg} \\
556 \mathrm{~kg}\end{array}$ & Paulus, unpublished & $\begin{array}{l}\mathrm{N}, \mathrm{C}, \\
\mathrm{D}, \mathrm{M}\end{array}$ \\
\hline 22 & 1994 & Watsonville & $\begin{array}{l}\text { MS } \\
12 \text { UC } \\
\text { cultivars/ } \\
\text { selections }\end{array}$ & $\begin{array}{l}917 \mathrm{~L} \\
\text { First NF }\end{array}$ & $\mathrm{NF}$ & --- & Shaw, unpublished & $\mathrm{N}$ \\
\hline 23 & 1994 & Watsonville & Selva & First $\mathrm{CP}^{\mathrm{x}}$ and $\mathrm{NF}$ & $\begin{array}{l}\mathrm{NF} \\
\mathrm{CP}\end{array}$ & $231 \mathrm{~kg}$ & Welch et al., 1995 & $\mathrm{~N}, \mathrm{C}$ \\
\hline 24 & 1995 & Irvine & Chandler & $\begin{array}{l}\text { Second CP } \\
\text { and NF crop }\end{array}$ & $\begin{array}{l}\mathrm{NF} \\
\mathrm{CP}\end{array}$ & $336 \mathrm{~kg}$ & Larson, unpublished & $\mathrm{N}, \mathrm{C}$ \\
\hline 25 & 1995 & Irvine & Chandler & Second NF & NF & --- & Larson, unpublished & $\mathrm{N}$ \\
\hline 26 & 1995 & Irvine & Chandler & Second CP & $\mathrm{CP}$ & $336 \mathrm{~kg}$ & Larson, unpublished & $\mathrm{C}$ \\
\hline 27 & 1995 & Irvine & Camarosa & Second NF & NF & --- & Larson, unpublished & $\mathrm{N}$ \\
\hline 28 & 1995 & Watsonville & Selva & Second CP and NF & $\begin{array}{l}\mathrm{NF} \\
\mathrm{CP}\end{array}$ & $336 \mathrm{~kg}$ & Larson, unpublished & $\mathrm{N}, \mathrm{C}$ \\
\hline 29 & 1995 & Watsonville & Selva & Second CP and NF & $\begin{array}{l}\mathrm{NF} \\
\mathrm{CP}\end{array}$ & $336 \mathrm{~kg}$ & Larson, unpublished & $\mathrm{N}, \mathrm{C}$ \\
\hline 30 & 1995 & Watsonville & Sunset & Second CP and NF & $\begin{array}{l}\mathrm{NF} \\
\mathrm{CP}\end{array}$ & $336 \mathrm{~kg}$ & Larson, unpublished & $\mathrm{N}, \mathrm{C}$ \\
\hline 31 & 1995 & Oxnard & Chandler & $\begin{array}{l}\text { First DP: } \mathrm{CP}^{\mathrm{x}}, \mathrm{CP}^{\mathrm{x}} \\
\text { and } \mathrm{NF}\end{array}$ & $\begin{array}{l}\mathrm{NF} \\
\mathrm{CP} \\
7 \mathrm{DP}: 3 \mathrm{CP}\end{array}$ & $\begin{array}{c}336 \mathrm{~kg} \\
539 \mathrm{~kg}\end{array}$ & Paulus, unpublished & $\begin{array}{l}\mathrm{N}, \mathrm{C}, \\
\mathrm{D}\end{array}$ \\
\hline 32 & 1995 & Watsonville & $\begin{array}{l}\text { nine UC } \\
\text { cultivars }\end{array}$ & Second NF & $\mathrm{NF}$ & --- & Shaw and Larson, 1996 & $\mathrm{~N}$ \\
\hline 33 & 1995 & Watsonville & $\begin{array}{l}\text { nine non-UC } \\
\text { cultivars }\end{array}$ & Second NF & NF & --- & Shaw and Larson, 1996 & $\mathrm{~N}$ \\
\hline 34 & 1995 & Watsonville & $\begin{array}{l}12 \text { UC } \\
\text { cultivars/ } \\
\text { selections }\end{array}$ & Second NF & NF & --- & Shaw, unpublished & $\mathrm{N}$ \\
\hline 35 & 1996 & Irvine & Camarosa & Third CP & $\mathrm{CP}$ & $336 \mathrm{~kg}$ & Larson, unpublished & $\mathrm{C}$ \\
\hline 36 & 1996 & Irvine & $\begin{array}{l}\text { Chandler, } \\
\text { Nur. trt.w } \\
=\text { low CP }\end{array}$ & Third CP & $\mathrm{CP}$ & $336 \mathrm{~kg}$ & Larson, unpublished & $\mathrm{C}$ \\
\hline 37 & 1996 & Irvine & $\begin{array}{l}\text { Chandler, } \\
\text { Nur. trt. } \\
=\text { low } \mathrm{CP}\end{array}$ & Third CP & $\mathrm{CP}$ & $336 \mathrm{~kg}$ & $\begin{array}{l}\text { Larson, unpublished } \\
\text { continued }\end{array}$ & $\begin{array}{l}\mathrm{C} \\
\text { tpage }\end{array}$ \\
\hline
\end{tabular}


Table 1. Continued.

\begin{tabular}{|c|c|c|c|c|c|c|c|c|}
\hline $\begin{array}{l}\text { Study } \\
\text { no. }\end{array}$ & $\begin{array}{l}\text { Test } \\
\text { year }\end{array}$ & Location & $\begin{array}{l}\text { Cultivars/ } \\
\text { selections }\end{array}$ & Crop & Treatment $^{2}$ & Rate/ha & Reference & $\begin{array}{l}\text { Study } \\
\text { set }^{y}\end{array}$ \\
\hline 39 & 1996 & Irvine & Camarosa & $\begin{array}{l}\text { First CP crop } \\
\text { after NF }\end{array}$ & $\mathrm{CP}$ & $168 \mathrm{~kg}$ & Larson, unpublished & $\mathrm{C}$ \\
\hline 40 & 1996 & Irvine & $\begin{array}{l}\text { Chandler, } \\
\text { Nur. trt." } \\
=\text { low } \mathrm{CP}\end{array}$ & $\begin{array}{l}\text { First CP crop } \\
\text { after NF }\end{array}$ & $\mathrm{CP}$ & $168 \mathrm{~kg}$ & Larson, unpublished & $\mathrm{C}$ \\
\hline 42 & 1996 & Irvine & $\begin{array}{l}\text { Chandler, } \\
\text { Nur. trt." } \\
=\mathrm{NF}\end{array}$ & $\begin{array}{l}\text { First CP crop } \\
\text { after NF }\end{array}$ & $\mathrm{CP}$ & $168 \mathrm{~kg}$ & Larson, unpublished & $\mathrm{C}$ \\
\hline 43 & 1996 & Irvine & Camarosa & Third NF & $\mathrm{NF}$ & --- & Larson, unpublished & $\mathrm{N}$ \\
\hline 44 & 1996 & Irvine & $\begin{array}{l}\text { Chandler, } \\
\text { Nur. trt. } \\
=\text { low } \mathrm{CP}\end{array}$ & Third NF & NF & --- & Larson, unpublished & $\mathrm{N}$ \\
\hline 46 & 1996 & Irvine & $\begin{array}{l}\text { Chandler, } \\
\text { Nur. trt." } \\
=\mathrm{NF}\end{array}$ & Third NF & NF & --- & Larson, unpublished & $\mathrm{N}$ \\
\hline 47 & 1996 & Irvine & Camarosa & $\begin{array}{l}\text { First } \mathrm{MS}^{\mathrm{x}} \text { crop } \\
\text { after NF }\end{array}$ & MS & $935 \mathrm{~L}$ & Larson, unpublished & M \\
\hline 48 & 1996 & Irvine & $\begin{array}{l}\text { Chandler, } \\
\text { Nur. trt." } \\
=\text { low CP }\end{array}$ & $\begin{array}{l}\text { First } \mathrm{MS}^{\mathrm{x}} \text { crop } \\
\text { after NF }\end{array}$ & MS & $935 \mathrm{~L}$ & Larson, unpublished & M \\
\hline 49 & 1996 & Irvine & $\begin{array}{l}\text { Chandler, } \\
\text { Nur. trt. } \\
=\text { high CP }\end{array}$ & $\begin{array}{l}\text { First } \mathrm{MS}^{\mathrm{x}} \text { crop } \\
\text { after NF }\end{array}$ & MS & $935 \mathrm{~L}$ & Larson, unpublished & M \\
\hline 50 & 1996 & Irvine & $\begin{array}{l}\text { Chandler, } \\
\text { Nur. trt." } \\
=\mathrm{NF}\end{array}$ & $\begin{array}{l}\text { First } \mathrm{MS}^{\mathrm{x}} \text { crop } \\
\text { after NF }\end{array}$ & MS & $935 \mathrm{~L}$ & Larson, unpublished & M \\
\hline 58 & 1996 & Oxnard & Chandler & $\begin{array}{l}\text { First DP:CP, CP } \\
\text { and NF crop }\end{array}$ & $\begin{array}{l}\mathrm{NF} \\
\mathrm{CP} \\
7 \mathrm{DP}: 3 \mathrm{CP}\end{array}$ & $\begin{array}{c}-\overline{-} \\
358 \mathrm{~kg} \\
389 \mathrm{~kg}\end{array}$ & Paulus, unpublished & $\mathrm{N}, \mathrm{C}, \mathrm{D}$ \\
\hline 59 & 1996 & Watsonville & $\begin{array}{l}12 \text { UC } \\
\text { cultivars/ } \\
\text { selections }\end{array}$ & Second NF & $\mathrm{NF}$ & --- & Shaw, unpublished & $\mathrm{N}$ \\
\hline 60 & 1996 & Watsonville & $\begin{array}{l}8 \text { UC } \\
\text { cultivars/ } \\
\text { selections }\end{array}$ & First CP & $\mathrm{CP}$ & $336 \mathrm{~kg}$ & Shaw, unpublished & $\mathrm{C}$ \\
\hline 61 & 1996 & Watsonville & $\begin{array}{l}8 \text { UC } \\
\text { cultivars/ } \\
\text { selections }\end{array}$ & First CP & $\mathrm{CP}$ & $168 \mathrm{~kg}$ & Shaw, unpublished & $\mathrm{C}$ \\
\hline 62 & 1996 & Watsonville & Selva & First $\mathrm{MS}^{\mathrm{x}}$ & MS & $468 \mathrm{~L}$ & Larson, unpublished & M \\
\hline 63 & 1996 & Watsonville & Selva & First $\mathrm{MS}^{\mathrm{x}}$ & MS & $935 \mathrm{~L}$ & Larson, unpublished & M \\
\hline 64 & 1997 & Watsonville & Selva & $\begin{array}{l}\text { First DP:CP, } \\
\text { Third NF }\end{array}$ & $\begin{array}{l}\text { NF } \\
65 \text { DP:35 CP }\end{array}$ & $\stackrel{---}{4} \mathrm{~kg}$ & Larson, unpublished & $\mathrm{N}, \mathrm{D}$ \\
\hline 65 & 1997 & Watsonville & $\begin{array}{l}5 \text { UC } \\
\text { cultivars/ } \\
\text { selections }\end{array}$ & $\begin{array}{l}\text { First DP:CP, } \\
\text { Third NF }\end{array}$ & $\begin{array}{l}\text { NF } \\
65 \text { DP:35 CP }\end{array}$ & $448 \mathrm{~kg}$ & Shaw, unpublished & $\mathrm{N}, \mathrm{D}$ \\
\hline 66 & 1997 & Irvine & Chandler & $\begin{array}{l}\text { First DP:CPx and } \\
\text { CP }^{\mathrm{x}} \text {, fourth NF }\end{array}$ & $\begin{array}{l}\mathrm{NF} \\
\mathrm{CP} \\
65 \mathrm{DP}: 35 \mathrm{CP}\end{array}$ & $\begin{array}{l}--- \\
336 \mathrm{~kg} \\
386 \mathrm{~kg}\end{array}$ & Larson, unpublished & N,C,D \\
\hline 67 & 1997 & Irvine & Camarosa & $\begin{array}{l}\text { First DP: } \mathrm{CP}^{\mathrm{x}}, \mathrm{CP}^{\mathrm{x}} \\
\text { and NF }\end{array}$ & $\begin{array}{l}\mathrm{NF} \\
\mathrm{CP} \\
4 \mathrm{DP}: 6 \mathrm{CP}\end{array}$ & $\begin{array}{r}-\overline{k g} \\
224 \mathrm{~kg} \\
392 \mathrm{~kg}\end{array}$ & Paulus et al., 1998 & $\mathrm{~N}, \mathrm{C}, \mathrm{D}$ \\
\hline
\end{tabular}

${ }^{\mathrm{z}}$ Mixtures of dichloropropene:chloropicrin were used at four rates. All trials were established using one of the treatments listed and $2 \mathrm{MB}: 1 \mathrm{CP}(\mathrm{wt}: \mathrm{wt})$ with a rate of $392 \mathrm{~kg} \cdot \mathrm{ha}^{-1}$; MB:CP rates differed for cases $21\left(427 \mathrm{~kg} \cdot \mathrm{ha} \mathrm{A}^{-1}\right), 31\left(405 \mathrm{~kg} \cdot \mathrm{ha}^{-1}\right), 58\left(383 \mathrm{~kg} \cdot \mathrm{ha}^{-1}\right), 67\left(57 \mathrm{MB}: 43 \mathrm{CP} @ 420 \mathrm{~kg} \cdot \mathrm{ha} a^{-1}\right)$. All MS applications were performed using a formulation with $33.4 \%$ a.i.

${ }^{\mathrm{y}}$ Studies labeled N, C, D, and $\mathrm{M}$ were used in comparisons between MB:CP and NF (N), CP(C), DP:CP (D), and MS (M) alternatives.

${ }^{x}$ Fumigants applied using bed application methods. MB treatments were applied using bed application methods to studies 1-8, 21, 23, 31, 66, and 67.

${ }^{w}$ Nur. trt. = nursery fumigation treatment; low CP is $168-224 \mathrm{~kg} \cdot \mathrm{ha}^{-1}$, high CP is $336-396 \mathrm{~kg} \cdot \mathrm{ha}^{-1}$; NF is nonfumigated. 
fumigation response for yield, and the overall unweighted increase in yield due to fumigation was $94.4 \%$.

The difference between yields for plants in fumigated and NF soils increased over the first three strawberry cultivation cycles. Fumigation with $\mathrm{MB}: \mathrm{CP}$ provided a standardized effects of $d_{+}=2.166 \pm 0.146(59.2 \%$ yield advantage) for 17 studies where one cycle of fumigation was skipped or soil not recently cropped in strawberry was used, $\mathrm{d}_{+}=3.000 \pm$ 0.143 (100.2\% advantage) for 19 studies where strawberries were cultivated for two cycles without MB:CP fumigation, and $\mathrm{d}_{+}=6.201 \pm$ 0.348 ( $148.4 \%$ yield advantage) in nine cases where strawberries were grown without soil fumigation for three or more cycles. Direct comparison of yields over cycles was not possible because the subsets of studies used different genotypes, harvest periods, etc. However, because nearly all the studies were conducted on ground that had been fumigated with MB:CP and cropped in strawberry for several prior cycles, the increase in $\mathrm{d}_{+}$, or the change in relative yield advantage reported here over cycles, can be attributed to the reduction of yield with repeated cultivation in NF soils, rather than increases in yield due to serial fumigation with MB:CP. Because the $95 \%$ confidence limits for these three effect size estimates do not overlap, these results indicate significant yield reduction at each of the first three NF cultivation cycles, and the minimum productivity expected without soil fumigation has likely not been reached in the NF soil environments evaluated to date.

Results for the 34 individual studies comparing MB:CP fumigation with $\mathrm{CP}$ alone are summarized in Table 3. Yields following fumigation with $\mathrm{MB}: \mathrm{CP}$ were larger than for $\mathrm{CP}$ fumigation in 28 of the 34 studies, and these differences were significant or highly significant in 12 of the 34 individual studies evaluated. Over all studies, the standardized effect was $d_{+}=0.751 \pm 0.087$ (95\% confidence: $0.581,0.921)$, indicating a significant yield advantage for combinations of MB:CP over $\mathrm{CP}$ alone, and the overall unweighted increase in yield due to fumigation was $9.6 \%$.

As a second step in evaluation of the $\mathrm{CP}$ alternative, the 34 studies were subdivided into a set of 23 studies where the rate of application was equal to or greater than 336 $\mathrm{kg} \cdot \mathrm{ha}^{-1} \mathrm{CP}$, and a set of 11 studies with rates between 121 and $224 \mathrm{~kg} \cdot \mathrm{ha}^{-1}$. At the higher application rate, the standardized effect was $\mathrm{d}_{+}$ $=0.437 \pm 0.114$ (unweighted increase $=6.8 \%$ ) whereas the lower application rate gave $\mathrm{d}_{+}=$ $1.190 \pm 0.134$ (unweighted increase $=15.4 \%$ ); 95\% confidence limits for these subset effect sizes did not overlap, demonstrating a statistically significant dependence on application rate.

As a final step in comparing these two alternatives, the 23 studies in the high application rate subset were further subdivided based on the number of strawberry cultivation cycles between the last MB:CP treatment and the current evaluation. Similar to the case comparing $\mathrm{MB}: \mathrm{CP}$ with $\mathrm{NF}$, the difference between yields for plants in soils fumigated with a $\mathrm{MB}: \mathrm{CP}$ mixture and soils treated with $\mathrm{CP}$

Table 2. Effects of soil fumigation with methyl bromide : chloropicrin (MB:CP) vs. nonfumigated (NF) control studies on yields (grams/plant) of strawberry in 45 studies.

\begin{tabular}{|c|c|c|c|c|c|c|c|c|c|}
\hline \multirow[b]{2}{*}{ Study } & \multirow[b]{2}{*}{ No. reps } & \multicolumn{2}{|c|}{ Treated } & \multicolumn{2}{|c|}{ Nontreated } & \multirow[b]{2}{*}{$\begin{array}{c}\text { Increase }^{\mathrm{z}} \\
(\%)\end{array}$} & \multirow[b]{2}{*}{$t^{\mathrm{y}}$} & \multirow[b]{2}{*}{$p^{\mathrm{y}}$} & \multirow[b]{2}{*}{$\mathrm{d}^{\mathrm{y}}$} \\
\hline & & $\begin{array}{c}\text { Mean } \\
\text { yield }\end{array}$ & SD & $\begin{array}{c}\text { Mean } \\
\text { yield }\end{array}$ & SD & & & & \\
\hline 1 & 5 & 1007 & 74 & 502 & 63 & 100.6 & 11.62 & $<0.001$ & 7.35 \\
\hline 2 & 6 & 992 & 177 & 390 & 104 & 154.4 & 7.18 & $<0.001$ & 4.15 \\
\hline 3 & 6 & 916 & 136 & 384 & 167 & 138.5 & 6.05 & $<0.001$ & 3.49 \\
\hline 4 & 5 & 1720 & 82 & 710 & 135 & 142.3 & 14.30 & $<0.001$ & 9.04 \\
\hline 5 & 6 & 1331 & 40 & 463 & 112 & 187.4 & 17.87 & $<0.001$ & 10.32 \\
\hline 6 & 6 & 1734 & 212 & 713 & 117 & 143.2 & 10.32 & $<0.001$ & 5.96 \\
\hline 7 & 5 & 1069 & 110 & 619 & 122 & 72.7 & 6.12 & $<0.001$ & 3.87 \\
\hline 9 & 24 & 1480 & 359 & 752 & 173 & 96.8 & 8.95 & $<0.001$ & 2.58 \\
\hline 10 & 24 & 833 & 218 & 476 & 154 & 75.0 & 6.55 & $<0.001$ & 1.895 \\
\hline 11 & 18 & 763 & 80 & 557 & 116 & 37.0 & 3.58 & 0.003 & 2.07 \\
\hline 12 & 18 & 772 & 64 & 535 & 95 & 44.3 & 5.07 & $<0.001$ & 2.92 \\
\hline 13 & 16 & 1239 & 62 & 529 & 46 & 134.2 & 36.79 & $<0.001$ & 13.01 \\
\hline 14 & 6 & 1704 & 44 & 1227 & 37 & 38.9 & 20.23 & $<0.001$ & 11.73 \\
\hline 15 & 24 & 1365 & 219 & 992 & 183 & 37.6 & 4.53 & 0.001 & 1.84 \\
\hline 16 & 6 & 1423 & 106 & 1112 & 101 & 28.0 & 5.20 & $<0.001$ & 3.00 \\
\hline 17 & 4 & 1493 & 177 & 1149 & 332 & 30.0 & 1.83 & 0.055 & 1.29 \\
\hline 18 & 4 & 1580 & 122 & 1328 & 233 & 19.0 & 1.92 & 0.048 & 1.36 \\
\hline 19 & 5 & 1343 & 169 & 1118 & 148 & 20.0 & 2.24 & 0.026 & 1.42 \\
\hline 20 & 5 & 1351 & 144 & 1227 & 390 & 10.1 & 0.67 & 0.259 & 0.42 \\
\hline 21 & 6 & 886 & 71 & 503 & 50 & 76.0 & 10.82 & $<0.001$ & 6.25 \\
\hline 22 & 24 & 1346 & 252 & 1017 & 227 & 32.4 & 4.84 & $<0.001$ & 1.40 \\
\hline 23 & 5 & 1643 & 78 & 1012 & 81 & 62.4 & 12.55 & $<0.001$ & 7.93 \\
\hline 24 & 12 & 1369 & 191 & 891 & 102 & 53.6 & 7.65 & $<0.001$ & 3.12 \\
\hline 26 & 12 & 1415 & 158 & 989 & 113 & 43.1 & 6.12 & $<0.001$ & 3.87 \\
\hline 27 & 16 & 1449 & 172 & 651 & 94 & 122.5 & 11.52 & $<0.001$ & 5.76 \\
\hline 28 & 4 & 1397 & 192 & 400 & 70 & 249.3 & 9.76 & $<0.001$ & 6.90 \\
\hline 29 & 4 & 1553 & 135 & 571 & 137 & 172.0 & 10.21 & $<0.001$ & 7.22 \\
\hline 30 & 8 & 1352 & 286 & 595 & 140 & 127.2 & 6.72 & $<0.001$ & 3.36 \\
\hline 31 & 4 & 655 & 65 & 353 & 42 & 85.4 & 7.75 & $<0.001$ & 5.48 \\
\hline 32 & 18 & 1296 & 355 & 733 & 345 & 76.8 & 4.83 & $<0.001$ & 1.61 \\
\hline 33 & 18 & 578 & 310 & 263 & 207 & 119.8 & 3.59 & $<0.001$ & 1.20 \\
\hline 34 & 24 & 1602 & 276 & 792 & 157 & 102.3 & 11.70 & $<0.001$ & 3.38 \\
\hline 35 & 12 & 1460 & 59 & 521 & 77 & 180.2 & 33.53 & $<0.001$ & 13.69 \\
\hline 46 & 12 & 1282 & 55 & 511 & 56 & 150.9 & 34.02 & $<0.001$ & 13.89 \\
\hline 51 & 18 & 1357 & 114 & 760 & 90 & 78.5 & 17.43 & $<0.001$ & 5.81 \\
\hline 52 & 18 & 1503 & 241 & 708 & 166 & 112.2 & 11.53 & $<0.001$ & 3.84 \\
\hline 55 & 12 & 1648 & 66 & 547 & 116 & 201.3 & 28.58 & $<0.001$ & 11.67 \\
\hline 56 & 8 & 1540 & 89 & 887 & 136 & 73.6 & 11.36 & $<0.001$ & 5.68 \\
\hline 57 & 4 & 1401 & 80 & 528 & 127 & 165.3 & 11.63 & $<0.001$ & 8.23 \\
\hline 58 & 6 & 871 & 56 & 546 & 36 & 59.6 & 11.89 & $<0.001$ & 6.86 \\
\hline 59 & 24 & 1627 & 125 & 1058 & 112 & 53.8 & 16.61 & $<0.001$ & 4.79 \\
\hline 64 & 36 & 1381 & 146 & 759 & 118 & 81.9 & 19.88 & $<0.001$ & 4.69 \\
\hline 65 & 10 & 1742 & 131 & 955 & 152 & 82.4 & 12.40 & $<0.001$ & 5.55 \\
\hline 66 & 12 & 994 & 88 & 409 & 47 & 143.0 & 20.3 & $<0.001$ & 8.29 \\
\hline 67 & 4 & 610 & 46 & 465 & 46 & 31.2 & 4.46 & $<0.001$ & 3.15 \\
\hline
\end{tabular}

${ }^{2}$ Unweighted percent increase in yield resulting from MB:CP treatment

${ }^{\mathrm{y}} t$ is Student's $t$ test value, $p$ is a one-tailed probability (requires $P<0.025$ for conventional significance), and $\mathrm{d}$ is the standardized effect size.

alone increased over the first three cultivation cycles: MB:CP fumigated soils provided a standardized effect of $\mathrm{d}_{+}=0.043 \pm 0.162$ (2.2\% yield advantage for MB:CP) for 12 studies where one cycle of $\mathrm{MB}: \mathrm{CP}$ fumigation was replaced by $\mathrm{CP}$ alone, $\mathrm{d}_{+}=0.588 \pm 0.174$ $(10.6 \%$ advantage for $\mathrm{MB}: \mathrm{CP})$ for seven studies where strawberries were cultivated for two cycles with high rates of $\mathrm{CP}$, and $\mathrm{d}_{+}=2.054 \pm$ $0.401(13.7 \%$ yield advantage for $\mathrm{MB}: \mathrm{CP})$ in four cases in which $\mathrm{CP}$ was used for three cycles. Although the initial yield decline after fumigation with high rates of $\mathrm{CP}$ was small, this appears largely the consequence of a carryover effect of MB:CP fumigation for a single cycle; even on soils fumigated with high rates of $\mathrm{CP}$ yield declined as a new equilibrium was approached after more than one strawberry production cycle.

Yields for MB:CP-treated soils exceeded those for DP:CP treated soils in nine of the ten individual studies reported here (Table 4), and individual differences were statistically significant in four of these nine cases. Over all 10 studies, the standardized effect was $\mathrm{d}_{+}=0.962$ \pm 0.162 (95\% confidence: $0.645,1.279$ ), indicating a significant yield advantage for combinations of MB:CP over DP:CP combinations; the overall unweighted increase in yield due to fumigation with MB:CP was $14.4 \%$. Performance of plants was statistically indistinguishable in soils fumigated with DP:CP mixtures and low rates of $\mathrm{CP}$ alone. Mixtures of DP:CP mixtures that use higher rates of $\mathrm{CP}$ might perform better on average, but there is no strong indication for this in the present set of studies.

Soil treatment with MS proved the least productive of the chemical alternatives tested, with yields for MB:CP treatments exceeding those for MS treated soils significantly in all eight individual studies (Table 5). Over all 
eight studies the standardized effect was $\mathrm{d}_{+}=$ $3.199 \pm 0.287$ (95\% confidence: $2.637,3.761$ ), indicating a significant yield advantage for combinations of $\mathrm{MB}: \mathrm{CP}$ over $\mathrm{MS}$, and the overall unweighted increase in yield due to fumigation with the current industry standard was $29.8 \%$. These results are consistent with the three studies eliminated because of insufficient data, where the average increase for MB:CP over MS was $29.0 \%$. In fact, the standardized effect for this comparison did not differ significantly from the overall comparison of MB:CP mixtures with $\mathrm{NF}\left(\mathrm{d}_{+}=2.874 \pm\right.$ 0.098), suggesting very little yield response following treatment with MS.

\section{Discussion}

The results of all meta-analyses are summarized in Table 6. For these studies, fumigation with a mixture of $\mathrm{MB}$ and $\mathrm{CP}$ increased yield significantly in comparison with any and all alternatives lacking MB. Mixtures of DP:CP that include a greater proportion of $\mathrm{CP}$ may provide a superior alternative to those tested here, although the deterioration of soil conditions for systems treated with high rates of $\mathrm{CP}$ alone over multiple cycles suggests that this likelihood is small. We conclude from this synthesis that there is no direct substitute for the current industry standard of preplant soil fumigation with mixtures of MB:CP. Furthermore, strawberry growers in California and other strawberry production locations with similar climate, using similar cultural tools, can expect yield reductions in excess of $10.5 \%$ because of changes in fruiting field fumigation practices alone if $\mathrm{MB}$ is eventually banned.

Yield reduction in the strawberry fruiting field is just one of many potential results of a regulatory elimination of $\mathrm{MB}$. Of immediate and quantifiable consequence is the use of fumigation alternatives in runner propagation nurseries. Fumigation with $\mathrm{MB}: \mathrm{CP}$ is practiced in such nurseries to enhance both plant production and quality (Larson and Shaw, 1995b) and to ensure a source of pest and disease free planting stock. Preliminary indications here are that use of planting stock from strawberry nurseries fumigated with realistic alternatives may reduce cumulative fruit yield substantially (Larson and Shaw, 1995b), even in the absence of lethal pathogens. Use of alternate fumigants will eventually impact the incidence of lethal pathogens in nursery and fruiting fields, and disease incidence may increase toward those levels experienced prior to widespread use of MB:CP (Wilhelm and Paulus, 1980).

Present-day cultural systems have evolved substantially during the past 50 years (Shaw, 1990; Voth and Bringhurst, 1990), and the trend has been toward an optimization relative to the superior soil environment provided by fumigation with MB:CP. Changes in fumigation practices that affect plant vigor and weed control can be expected to mandate changes in horticultural systems that in themselves will modify the magnitude and timing of strawberry fruit production. For example, alternatives deficient in weed control may require use

Table 3. Effects of soil fumigation with methyl bromide:chloropicrin (MB:CP) vs. chloropicrin $(\mathrm{CP})$ on yields (grams/plant) of strawberry in 34 studies.

\begin{tabular}{|c|c|c|c|c|c|c|c|c|c|}
\hline \multirow[b]{2}{*}{ Study } & \multirow[b]{2}{*}{$\begin{array}{l}\text { No. } \\
\text { reps }\end{array}$} & \multicolumn{2}{|c|}{ MB:CP treated } & \multicolumn{2}{|c|}{$\mathrm{CP}$ treated } & \multirow[b]{2}{*}{$\begin{array}{c}\text { Increase }^{2} \\
(\%)\end{array}$} & \multirow[b]{2}{*}{$t^{y}$} & \multirow[b]{2}{*}{$p^{\mathrm{y}}$} & \multirow[b]{2}{*}{$\mathrm{d}^{\mathrm{y}}$} \\
\hline & & $\begin{array}{l}\text { Mean } \\
\text { yield }\end{array}$ & SD & $\begin{array}{l}\text { Mean } \\
\text { yield }\end{array}$ & SD & & & & \\
\hline 11 & 18 & 763 & 80 & 768 & 84 & -0.0 & -0.10 & 0.538 & -0.06 \\
\hline 12 & 18 & 772 & 64 & 780 & 83 & -1.0 & -0.18 & 0.569 & -0.11 \\
\hline 15 & 24 & 1365 & 219 & 1283 & 286 & 6.4 & 0.79 & 0.219 & 0.32 \\
\hline 16 & 6 & 1423 & 106 & 1233 & 252 & 15.4 & 1.70 & 0.060 & \\
\hline 17 & 4 & 1493 & 177 & 1477 & 162 & 1.2 & 0.13 & 0.451 & 0.0 \\
\hline 18 & 4 & 1580 & 122 & 1528 & 76 & 3.4 & 0.72 & 0.248 & 0.51 \\
\hline 19 & 5 & 1343 & 169 & 1371 & 390 & -2.0 & -0.15 & 0.558 & -0.09 \\
\hline 20 & 5 & 1351 & 144 & 1470 & 206 & -8.1 & -1.06 & 0.842 & -0.67 \\
\hline 23 & 5 & 1643 & 78 & 1502 & 141 & 9.4 & 1.96 & 0.043 & 1.24 \\
\hline 24 & 12 & 1369 & 191 & 1227 & 110 & 11.6 & 2.23 & 0.018 & 0.91 \\
\hline 26 & 12 & 1415 & 158 & 1316 & 125 & 7.5 & 1.70 & 0.052 & 0.6 \\
\hline 27 & 6 & 886 & 71 & 823 & 70 & 7.7 & 1.56 & 0.075 & 0.9 \\
\hline 28 & 4 & 1397 & 192 & 1303 & 77 & 7.2 & 0.91 & 0.197 & 0.64 \\
\hline 29 & 4 & 1553 & 135 & 1270 & 234 & 22.3 & 2.10 & 0.040 & 1.48 \\
\hline 30 & 8 & 1352 & 286 & 1304 & 381 & 3.7 & 0.28 & 0.392 & \\
\hline 31 & 4 & 655 & 65 & 598 & 96 & 9.3 & 0.95 & 0.190 & \\
\hline 35 & 12,6 & 1648 & 66 & 1409 & 99 & 17.0 & 4.92 & $<0.001$ & 2.84 \\
\hline 36 & 4,2 & 1401 & 80 & 1267 & 205 & 10.6 & 0.86 & 0.250 & 0.86 \\
\hline 37 & 12,6 & 1460 & 59 & 1228 & 96 & 18.9 & 5.04 & $<0.001$ & 2.91 \\
\hline 38 & 12,6 & 1282 & 55 & 1185 & 74 & 8.2 & 2.58 & 0.011 & 1.49 \\
\hline 39 & 12 & 1648 & 66 & 1235 & 100 & 33.4 & 11.94 & $<0.001$ & 4.87 \\
\hline 40 & 4 & 1401 & 80 & 1209 & 303 & 15.9 & 1.23 & 0.143 & 0.87 \\
\hline 41 & 12 & 1460 & 59 & 1243 & 63 & 17.5 & 8.71 & $<0.001$ & 3.56 \\
\hline 42 & 12 & 1282 & 55 & 1068 & 52 & 20.0 & 9.79 & $<0.001$ & 4.00 \\
\hline 53 & 18 & 1358 & 114 & 1479 & 140 & -8.2 & -2.84 & 0.999 & -0.95 \\
\hline 54 & 18 & 1358 & 114 & 1168 & 125 & 16.3 & 4.76 & $<0.001$ & 1.59 \\
\hline 55 & 10 & 1540 & 90 & 1568 & 158 & -1.7 & -0.48 & 0.820 & -0.22 \\
\hline 57 & 10 & 1540 & 90 & 1316 & 138 & 17.0 & 4.29 & $<0.001$ & 1.92 \\
\hline 58 & 6 & 871 & 56 & 848 & 63 & 2.7 & 0.67 & 0.259 & 0.39 \\
\hline 59 & 8 & 1627 & 125 & 1454 & 262 & 11.9 & 1.69 & 0.057 & 0.84 \\
\hline 60 & 8 & 1627 & 125 & 1340 & 151 & 21.4 & 4.14 & $<0.001$ & 2.07 \\
\hline 64 & 36 & 1381 & 146 & 1204 & 176 & 14.7 & 4.64 & $<0.001$ & 1.09 \\
\hline 66 & 6 & 994 & 88 & 982 & 67 & 1.2 & 0.27 & 0.397 & 0.15 \\
\hline 67 & 4 & 610 & 46 & 531 & 46 & 14.9 & 2.43 & 0.025 & 1.72 \\
\hline
\end{tabular}

${ }^{2}$ Unweighted percent increase in yield for the MB:CP treatment over the CP treatment group.

${ }^{y} t$ is Student's $t$ test value, $p$ is a one-tailed probability (requires $P<0.025$ for conventional significance), and $d$ is the standardized effect size.

Table 4. Effects of soil fumigation with methyl bromide:chloropicrin (MB:CP) vs. dichloropropenechloropicrin (DP:CP) on yields (grams/plant) of strawberry in 10 studies.

\begin{tabular}{|c|c|c|c|c|c|c|c|c|c|}
\hline \multirow[b]{2}{*}{ Study } & \multirow[b]{2}{*}{$\begin{array}{l}\text { No. } \\
\text { reps }\end{array}$} & \multicolumn{2}{|c|}{ MB:CP treated } & \multicolumn{2}{|c|}{ DP:CP treated } & \multirow[b]{2}{*}{$\begin{array}{c}\text { Percent } \\
\text { increase }^{z}\end{array}$} & \multirow[b]{2}{*}{$t^{y}$} & \multirow[b]{2}{*}{$\mathrm{p}^{\mathrm{y}}$} & \multirow[b]{2}{*}{$\mathrm{d}^{\mathrm{y}}$} \\
\hline & & $\begin{array}{l}\text { Mean } \\
\text { yield }\end{array}$ & SD & $\begin{array}{l}\text { Mean } \\
\text { yield }\end{array}$ & SD & & & & \\
\hline 2 & 6 & 992 & 177 & 856 & 109 & 15.9 & 1.60 & 0.070 & 0.93 \\
\hline 5 & 6 & 1331 & 40 & 1046 & 55 & 27.2 & 10.27 & $<0.001$ & 5.93 \\
\hline 7 & 5 & 1096 & 110 & 687 & 62 & 59.5 & 6.76 & $<0.001$ & 4.28 \\
\hline 21 & 6 & 886 & 71 & 914 & 48 & -2.9 & -0.78 & 0.727 & -0.45 \\
\hline 31 & 4 & 655 & 65 & 647 & 54 & 1.0 & 0.15 & 0.443 & 0.11 \\
\hline 58 & 6 & 871 & 56 & 836 & 11 & 4.3 & 1.52 & 0.077 & 0.88 \\
\hline 64 & 36 & 1381 & 146 & 1180 & 185 & 17.0 & 5.12 & $<0.001$ & 1.21 \\
\hline 65 & 10 & 1742 & 131 & 1489 & 141 & 17.0 & 4.16 & $<0.001$ & 1.86 \\
\hline 66 & 6 & 994 & 88 & 981 & 97 & 1.3 & 0.37 & 0.355 & 0.15 \\
\hline 67 & 4 & 610 & 46 & 591 & 46 & 3.2 & 0.58 & 0.291 & 0.41 \\
\hline
\end{tabular}

${ }^{2}$ Unweighted percent increase in yield for the MB:CP treatment over the DP:CP treatment group.

${ }^{y} t$ is Student's $t$ test value, $p$ is a one-tailed probability (requires $P<0.025$ for conventional significance), and $\mathrm{d}$ is the standardized effect size.

of colored plastic mulches which will delay fruiting and reduce total yield. Furthermore, changes in both soil fumigation environment and cultural practice affect fruit quality (Larson and Shaw, 1995a), which will ultimately determine the value of the crop produced. The consequences of these secondary effects must be quantified and their outcomes considered in evaluating the ultimate impact of $\mathrm{MB}$ regulatory policies on strawberry productivity.

\section{Literature Cited}

Faxon, G. 1997. Annu. Rpt., Processing Strawberry Advisory Board of California, Watsonville.

Hedges, L.V. and I. Olkin. 1985. Statistical methods for meta analysis. Academic Press, Orlando, Fla.

Himelrick, D.G. and W.A. Dozier, Jr. 1991. Soil fumigation and soil solarization in strawberry production. Adv. Strawberry Prod. 10:12-28.

Larson, K.D. and Sterrett, J. 1994. Soil fumigation 
Table 5. Effects of soil fumigation with methyl bromide:chloropicrin (MB:CP) vs. metam sodium (MS) 153-305 L.ha ${ }^{-1}$ a.i. on yields (grams/plant) of strawberry in eight studies.

\begin{tabular}{|c|c|c|c|c|c|c|c|c|c|}
\hline \multirow[b]{2}{*}{ Study } & \multirow[b]{2}{*}{$\begin{array}{l}\text { No. } \\
\text { reps }\end{array}$} & \multicolumn{2}{|c|}{ MB:CP treated } & \multicolumn{2}{|c|}{ MS treated } & \multirow[b]{2}{*}{$\begin{array}{c}\text { Percent } \\
\text { increase }^{z}\end{array}$} & \multirow[b]{2}{*}{$t^{\mathrm{y}}$} & \multirow[b]{2}{*}{$p^{\mathrm{y}}$} & \multirow[b]{2}{*}{$\mathrm{d}^{\mathrm{y}}$} \\
\hline & & $\begin{array}{r}\text { Mean } \\
\text { yield }\end{array}$ & SD & $\begin{array}{l}\text { Mean } \\
\text { yield }\end{array}$ & SD & & & & \\
\hline 8 & 3 & 2126 & 47 & 1641 & 130 & 29.6 & 6.08 & 0.002 & 4.96 \\
\hline 21 & 6 & 886 & 71 & 667 & 94 & 32.8 & 4.55 & 0.001 & 2.63 \\
\hline 47 & 12 & 1648 & 66 & 1241 & 67 & 32.8 & 14.99 & $<0.001$ & 6.12 \\
\hline 48 & 4 & 1401 & 80 & 1184 & 119 & 18.3 & 3.03 & 0.012 & 2.14 \\
\hline 49 & 12 & 1460 & 59 & 1270 & 51 & 15.0 & 8.44 & $<0.001$ & 3.44 \\
\hline 50 & 12 & 1282 & 55 & 1136 & 62 & 12.9 & 6.10 & $<0.001$ & 2.49 \\
\hline 62 & 8,5 & 1540 & 89 & 982 & 182 & 56.8 & 6.16 & $<0.001$ & 3.90 \\
\hline 63 & 8,5 & 1540 & 89 & 1100 & 190 & 40.0 & 4.69 & $<0.001$ & 2.97 \\
\hline
\end{tabular}

${ }^{\mathrm{z}}$ Unweighted percent increase in yield for the MB:CP treatment over the MS control group.

${ }^{\mathrm{y}} t$ is Student's $t$ test value, $p$ is a one-tailed probability (requires $P<0.025$ for conventional significance), and $\mathrm{d}$ is the standardized effect size.

Table 6. Summary of meta-analysis comparisons presented in the text.

\begin{tabular}{lrrr}
\hline \hline & No. & & $\begin{array}{c}\text { Increase with } \\
\text { MB:CP }(\%)\end{array}$ \\
Comparison & studies & $\mathrm{d}_{+}( \pm \mathrm{SE})$ & MB \\
\hline Methyl bromide:chloropicrin vs. nonfumigated & 45 & $2.874 \pm 0.098$ & 94.4 \\
$\quad$ First nonfumigated cycle & 17 & $2.166 \pm 0.146$ & 59.2 \\
$\quad$ Second nonfumigated cycle & 19 & $3.000 \pm 0.143$ & 100.2 \\
$\quad$ Third nonfumigated cycle & 9 & $6.201 \pm 0.348$ & 148.4 \\
Methyl bromide:chloropicrin vs. chloropicrin & 34 & $0.751 \pm 0.087$ & 9.6 \\
$\quad$ High rate & 23 & $0.437 \pm 0.114$ & 6.8 \\
$\quad$ Moderate rate & 11 & $1.190 \pm 0.134$ & 15.4 \\
$\quad$ First chloropicrin cycle (high rate) & 12 & $0.043 \pm 0.162$ & 2.2 \\
$\quad$ Second chloropirin cycle (high rate) & 7 & $0.588 \pm 0.174$ & 10.6 \\
$\quad$ Third chloropicrin cycle (high rate) & 4 & $2.054 \pm 0.401$ & 13.7 \\
Methyl bromide:chloropicrin vs. dichloropropene:chloropicrin & 10 & $0.962 \pm 0.162$ & 14.4 \\
Methyl bromide:chloropicrin vs. metam sodium & 8 & $3.199 \pm 0.287$ & 29.8 \\
\hline
\end{tabular}

studies at Univ. of California South Coast Res. and Ext. Cen., 1992-93. The Pink Sheet: California Strawberry Commission Strawberry News Bul. 94-10, 13 Dec. 1994.

Larson, K.D. and Shaw, D.V. 1995. Nursery and fruiting field fumigation treatments and yield of Chandler. The Pink Sheet: California Strawberry Commission Strawberry News Bul. 96-3, 5 Feb. 1996

Larson, K.D. and Shaw, D.V. 1995a. Relative performance of strawberry genotypes in fumigated and nonfumigated soil. J. Amer. Soc. Hort. Sci. 120:274-277.

Larson, K.D. and Shaw, D.V. 1995b. Strawberry nursery soil fumigation and runner plant production. HortScience 30:236-237. its applications in horticultural science. HortScience 30:1343-1348.

Paulus, A.O., M. Vilchez, and M. Coffee. 1998. Alternatives to methyl bromide for soil fumigation. The Pink Sheet: California Strawberry
Olkin, I. and D.V. Shaw. 1995. Meta-analysis and
Commission Strawberry News Bul. 98-1, 8 Jan. 1998.

Shaw, Douglas V. 1990. Strawberries in America: Introduction to the symposium. HortScience 25:868.

Shaw, Douglas V. and K.D. Larson. 1996. Relative performance of strawberry cultivars from California and other North American sources in fumigated and nonfumigated soils. J. Amer. Soc. Hort. Sci. 121:764-767.

Voth, V. and R.S. Bringhurst. 1990. Culture and physiological manipulation of strawberries. HortScience 25:889-892.

Walsh, D., F. Zalom, D.V. Shaw, and N. Welch. 1996. Strawberry cultivar, soil fumigation, spidermite, inter-relationships. The Pink Sheet: California Strawberry Commission Strawberry News Bul. 96-4, 7 Feb. 1996.

Welch, N.C., W.D. Gubler, C. Pickle, and D. Walsh. 1990. Soil fumigation: Alternatives to methyl bromide chloropicrin, p. 92-94. In: Annu. Strawberry Res. Rpt., 1990. California Strawberry Commission.

Welch, N.C., W.D. Gubler, C. Pickle, and D. Walsh. 1991. Soil fumigation: Alternatives to methyl bromide chloropicrin, p. 97-100. In: Annu. Strawberry Res. Rpt., 1990. California Strawberry Commission.

Welch, N.C., W.D. Gubler, C. Pickle, and D. Walsh. 1995. Soil fumigation experiments in strawberries in the central coast district of California. The Pink Sheet: California Strawberry Commission Strawberry News Bul., 5 Feb. 1995.

Wilhelm, S. and E.C. Koch. 1956. Verticillium wilt controlled. California Agr. 10:3,14.

Wilhelm, S., R.C. Storkan, and J.E. Sagen. 1961. Verticillium wilt of strawberry controlled by fumigation of soil with chloropicrin and chloropicrin-methyl bromide mixtures. Phytopathology 51:744-748.

Wilhelm, S., R.C. Storkan, and J.M. Wilhelm. 1974. Preplant soil fumigation with methyl bromidechloropicrin mixtures for control of soil-borne diseases of strawberry-A summary of fifteen years of development. Agr. Environ. 1:227-236.

Wilhelm, S. and A.O. Paulus. 1980. How soil fumigation benefits the California strawberry industry. Plant Dis. 64:264-270. 